\title{
SIMPLE THEORETICAL ANALYSIS OF THE PHOTOEMISSION FROM CARBON NANOTUBES AND QUANTUM WIRES OF II-VI MATERIALS
}

\author{
Banerjee.S.N $\mathbf{N}^{1}$ and Ghatak .K.P ${ }^{2}$ \\ 'D.R.D.O., P.O. Chandipur, Balasore, Orissa. \\ ${ }^{2}$ Department of Electronic Science, University of Calcutta, India \\ E-mail : 1drsnbano@rediffmail.com
}

\begin{abstract}
We study the photoemission from $(n, n)$ and $(n, 0)$ carbon nanotubes (CNTs) and quantum wires $(\mathrm{QWs})$ of II-VI materials, considering the anisotropies of the effective electron masses, and the crystal field splitting of the later, within the k.p formalism. It has been found taking QWs of CdS, that the photoemission exhibits increasing plateaus as functions of increasing carrier concentration and incident photon energy. In case of CNTs, the photoemission is an increasing oscillatory function of both the said variables and is several times greater than that of the QWs of II-VI materials which is important from experimental point of view.
\end{abstract}

Key words: Carbon nanotubes, Photo emission, Guantom wires.

\section{INTRODUCTION}

In recent years, with the advent of modern lithography techniques [1], low-dimensional structures such as, ultrathin films, quantum wires (QWs) and quantum dots (QDs) have, in the last few years, attracted much attention not only for their potential in uncovering new phenomena in nano-photonics but also for novel technological applications. In QWs, the restriction of the motion of the carriers in the direction normal to the film (say, the zdirection) may be viewed as carrier confinement in an infinitely deep one dimensional (1D) square potential well, leading to quantization (known as quantum size effect (QSE)) of the wave vector, allowing 2D electron transport parallel to the surface representing new physical features not exhibited in bulk semiconductors [2]. As the dimension of the quantum well increases from $1 \mathrm{D}$ to $3 \mathrm{D}$, the degree of freedom of the free carriers, decreases drastically and the density-of-states function is changed from a stepped cumulative one to the Dirac's delta function [3].

In this context, it should be noted that the II-VI compounds find extensive applications in infrared imaging, ultra high speed bipolar transistors, fiber optic communications [4], advanced microwave devices together with the fact that these materials possess the appropriate direct gap to produce light emitting diodes and lasers. The carrier energy spectra in II-VI materials are defined by the Hopfield model [5], where the splitting of the two-spin states by the spin-orbit coupling and the crystalline field has been taken into account. It is well known that the carbon nanotubes (CNTs) are used as molecular QWs, CNT transistors, CNT FETs, field emission devices [6], etc. The one dimensional conduction in CNTs occur without any quantization in other two directions of the motions of the electrons where as in QWs, the said directions are quantized and electrons are allowed to move only in the single free direction.

The low-dimensional hetero-structures are widely investigated because of the enhancement of the carrier mobility which affects significantly the speed of performance of the electron devices at the device terminals of modern switching transistors [7]. These properties make such structures suitable for applications in QW waveguides, hetero-junction FETs, high-speed digital networks, high-frequency microwave circuits and other devices. Although many new effects associated with quantum confinement have already been reported, the interest for further research for such materials is becoming increasingly important. One such significant property is the photoemission and from the literature it appears to be the photoemission from CNTs and II-VI degenerate materials which has been relatively less studied under the quantization of band states. Therefore, it would be of interest to study the photoemission from such two different types of 1D structure. In Section 2.1 of the theoretical background, we shall study the photoemission from QWs of II-VI materials in accordance with the Hopfield model, where the splitting of the two-spin states by the spin-orbit coupling and the crystalline field has been taken into account. In section 2.2 , we shall study the photoemission form $(n, n)$ and $(n, 0)$ carbon nanotubes. In section 3 , we shall present the results and discussion.

\section{THEORETICAL BACKGROUND}

A. Formulation of the photoemission from QWs of II-VI materials

The carrier dispersion relation in bulk specimens of II- 
VI materials in accordance with Hopfield model can be expressed as [5]

$$
E=a_{0} k_{s}^{2}+b_{0} k_{z}^{2} \pm c_{0} k_{s}
$$

where $a_{0}=\mathrm{h}^{2} / 2 m_{\perp}^{*}, \mathrm{~h}=h / 2 \pi, h$ is the Planck's constant, $k^{2}=k_{s}^{2}+k_{z}^{2}, k_{s}^{2}=k_{x}^{2}+k_{y}^{2}, b_{0}=\mathrm{h}^{2} / 2 m_{\mathrm{p}}^{*}, c_{0}$ represents the splitting of the two-spin states by the spin orbit coupling and the crystalline field and $\mathrm{m}^{*}{ }_{\mathrm{p}}$ and $\mathrm{m}^{*}{ }_{\perp}$ are the effective masses of the electron at the edge of the conduction band parallel and perpendicular to the direction of the $\mathbf{z}$ axis, respectively. In the presence of the size quantization along $y$ and z-direction, Eqn. (1) assumes the form

$k_{x}^{2}=\phi\left(n_{y}, n_{z}, E\right)$

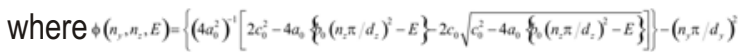

$n_{y}(=1,2,3, \ldots), n_{z}(=1,2,3, \ldots), d_{y}$ and $d_{z}$ are the size quantum numbers and the nano-thicknesses along the $y$ and z-direction respectively.

The sub band energies are given by

$E_{n_{z}}=\left(\mathrm{h}^{2} / 2 m_{\mathrm{P}}^{*}\right)\left(n_{z} \pi / d_{z}\right)^{2}$

The emission condition can be written as

$E_{n_{z_{\min }}}+h v \geq W$

where $h_{v}$ is the energy of the incident photon along z-axis and $W$ is the electron affinity and $E_{n z m i n}$ can be expressed through the Eq. (3) as

$E_{n_{z_{\text {int }}}}=\left(\mathrm{h}^{2} / 2 m_{\mathrm{p}}^{*}\right)\left(n_{z_{\text {mi }}} \pi / d_{z}\right)^{2}$

in which $n_{n z \min }$ is the minimum quantized level from which the photoemission occurs.

The total photoelectric current from QWs of II-VI materials $\left(J_{10}\right)$ can be written as

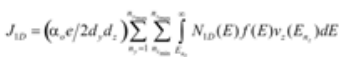

where $a_{0}$ is the probability of photon absorption, $e$ is the electron charge and $N_{1 D}(E)$ is the $1 D$ density-of-states function per subband, $f(E)$ is the Fermi-Dirac occupation probability factor, $\left.v_{z}\left(E_{n_{2}}\right)^{[}=\mathrm{h}^{-1}(\partial E / \partial k)_{E-E_{z_{1}}}\right]$ is the velocity of the electron in the sub band, the factor originates owing to the fact that only half of the electron will migrate towards the surface and escape [8] and can be determined from the equation

$n_{z_{\min }} \geq\left(d z \sqrt{2 m_{\mathrm{P}}^{*}} / \pi \mathrm{h}\right) \sqrt{W-h v}$

The $v_{z}\left(E_{n z}\right)$ in this case can be expressed as

$v_{z}\left(E_{n_{z}}\right)=\sqrt{2 E_{n_{z}} / m_{\mathrm{p}}^{*}}$

Thus, it appears that, the formulation of $J_{1 D}$ requires an expression $N_{1 N}(E)$ of which can be written in this case as
$N_{1 D}(E)=[2 / \pi]\left(\partial k_{x} / \partial E\right)$

Using Eqn. (2), (8) and (9), the 1D photoemission in this case can thus be expressed following Eqn. (6) as

$J_{1 D}=\left(\alpha_{o} e h \pi / d_{y} d_{z}^{2} m_{p}^{*}\right) \sum_{n=1}^{n} \sum_{n_{z}=0}^{n}\left[n_{z} \sqrt{\phi\left(n_{y}, n_{z}, E_{F}\right)}\right]$

where $E_{F}$ is the Fermi energy as measured from the edge of the conduction band in the vertically upward direction, $k_{B}$ is the Boltzmann's constant and $T$ is the temperature. The investigation of $J_{10}$ requires an expression electron statistics, which can be written as

$n_{1 D}=\sum_{n, 1}^{n} \sum_{n_{2}=1}^{\infty} \int_{E_{\infty}}^{\infty} N_{1 D}(E) f(E) d E$

Using Eqn. (9) and (11), we get

$n_{1 D}=\frac{2}{\pi} \sum_{n,=1}^{n_{n, n}} \sum_{n, z}^{n_{\text {non }}}\left[\sqrt{\phi\left(n_{y}, n_{z}, E_{F}\right)}\right]$

$B$. Formulation of the photoemission from $(n, n)$ and $(n, 0)$ carbon nanotubes

For the $(n, n)$ and $(n, 0)$ tubes, the respective energy dispersion relations are given by [9]

$E_{m}\left(k_{y}\right)= \pm \sqrt{1+4 \cos (m \pi / n) \cos \left(k_{y} \rho / 2\right)+4 \cos ^{2}\left(k_{y} \rho / 2\right)}, \quad-\pi / \rho<k_{y}<\pi / \rho$

and

$E_{m}\left(k_{x}\right)= \pm \sqrt{1+4 \cos \left(\sqrt{3} k_{x} \rho / n\right) \cos (n \pi / n)+4 \cos ^{2}(n \pi / n)}, \quad-\pi / \sqrt{3} \rho<k_{x}<\pi / \sqrt{3} \rho$

where $t(=-0.9 \mathrm{eV})$ is the tight binding constant, $\rho$ is the length of the basis vector for the lattice [9]. Using Eqns. (13) and (14), the electron concentration per unit length, can be written as,

$n_{1 D}=(4 / \pi \rho)\left[\bar{A}_{1}\left(E_{F}, m, n\right)\right]$

and

$n_{1 D}=(4 / \rho \pi \sqrt{3})\left[\bar{A}_{2}\left(E_{F}, m, n\right)\right]$

where, $\left.\bar{A}_{1}\left(E_{F}, m, n\right)=\cos ^{-1}\left[\left((A)^{2}-1+\left(E_{F} / t\right)^{2}\right)^{2}-\bar{A}\right) / 2\right], \bar{A}=\cos (n \pi / n)$ and.

$\bar{A}_{2}\left(E_{F}, m, n\right)=\cos ^{-1}\left[(4 \bar{A})^{-1}\left\{\left(E_{F} / t\right)^{2}-1-4(\bar{A})^{2}\right\}\right]$

Thus, the photoemission current for $(n, n)$ nanotubes assumes the form

$I_{1 D_{n, n}}=\left(2 \alpha_{0} e / \pi \mathrm{h}\right) \int_{E_{10}}^{\infty} f(E) d E$

where. $E_{10}=\left[ \pm t(5+4(\bar{A}))^{1 / 2}\right]+W-l N$

So that, from Eqn. (17), we get,

$I_{1 D_{n, n}}=\left(2 \alpha_{0} e k_{B} T / \pi \hbar\right) F_{0}\left[\xi_{1 \pm}\right]$

where $\xi_{1 \pm}=\left(k_{B} T\right)^{-1}\left[E_{F}-\left[\left[ \pm t(5+4(\bar{A}))^{1 / 2}\right]+W-l_{N}\right]\right]$.

Similarly, for $(n, 0)$ tubes, we can write, 
$I_{1 D_{n, 0}}=\left(2 \alpha_{0} e k_{B} T / \pi \mathrm{h}\right) F_{0}\left[\xi_{2 \pm}\right]$

where $\quad \xi_{2 \pm}=\left(k_{B} T\right)^{-1}\left[E_{F}-[[ \pm t(1+2(\bar{A}))]+W-l N]\right]$.

\section{RESULTS AND DISCUSSION}

Using Eqns. (10) and (12), we have plotted in Fig. 1 the 1D photoemission as a function of carrier concentration per unit length from $\mathrm{QWs}$ of CdS for which, $\mathrm{m}_{\mathrm{p}}^{*}=0.7 \mathrm{~m}_{0}$, $\mathrm{m}^{*}=1.5 \mathrm{~m}_{0}$ and $\mathrm{c}_{0}=1.4 \times 10^{-10}{ }_{\mathrm{e}} \mathrm{V}_{\mathrm{m}}$ at $T=4.2 \mathrm{~K}[10]$. The plot (b) refers to the $\mathrm{QWs}$ of $\mathrm{CdS}$ with $\mathrm{c}_{0}=0$ for the purpose of assessing the influence of the splitting of the two-spin states by the spin orbit coupling and the crystalline field. Using Eqns. (15), (18) and (16), (19), and taking the energy spectrum constants as defined in [9], we have plotted in the same figure, the photoemission from $(n, n)$ and $(n, 0)$ carbon nanotubes for the purpose of relative comparison respectively. In Fig. 2, we have plotted the photoemission as a function of incident photon intensity for all the cases of Fig. 1, which reflects that the photoemission depends strongly on the carrier concentration and the incident light intensity. For incident photon energies close to the electron affinity, the photoemission increases with increasing degeneracy with different numerical magnitudes. For $\mathrm{QWs}$, of $\mathrm{II}-\mathrm{VI}$ materials, the electrons create a net emission current comprising a number of ladder-like steps. It appears that the photocurrent from QWs of CdS exhibit greater numerical magnitudes in the presence of $\mathrm{C}_{0}$. It appears from both the figures that the numerical values of the photoemission from CNTs are the greatest together with the fact the photoemission exhibits composite sinusoidal unusual variations as compared with the QWs of II-VI materials and these types of oscillations are the characteristic features of carbon nanotubes. We also observed that the photoemission from QWs of II-VI materials exhibits increasing plateaus as a function of increasing incident photon energy, which is important from an experimental point of view, analogous to the same type of plateaus, which have been observed in quantum Hall effect in the variation of quantizing magnetic field. Moreover, the photoemissions from QWs can become several orders of magnitude larger than of bulk specimens of the same materials, which is also a direct signature of quantum confinement. This oscillatory dependence will be less and less prominent with increasing $d_{z}$ and ultimately, for bulk specimens, the photoemission will be found to increase continuously with increasing electron concentration in a non-oscillatory manner. The appearance of the humps of the figures is due to the redistribution of the electrons among the quantized energy levels when the size quantum number corresponding to the highest occupied level changes from one fixed value to the others. Consequently, the crossing of the Fermi level by the size-quantized levels in QWs would have much greater impact on the redistribution of the electrons among the allowed levels, as compared to that found for their bulk specimens. It is worth remarking that the influence of the energy band models on the photoemissions from various types of QWs and QDs of non-linear optical, IV-VI and stressed materials can also be assessed from our present work. We have not considered other types of compounds in order to keep the presentation brief. With different sets of energy band parameters, we shall get different numerical values of the photoemissions, though, the nature of variations of the photoemissions as shown here would be similar for the other types of materials and the simplified analysis of this paper exhibits the basic qualitative features of the photoemission from such compounds. If the direction normal to the film was taken differently from that as assumed in this work, the expressions for the photoemissions in QWs of II-VI materials would be different analytically, since the basic dispersion law as given by Eqn. (2) is anisotropic. Finally, it may be noted that the basic aim of this article is not solely to demonstrate the photoemission from two such completely different types of $1 \mathrm{D}$ structure, i.e., QWs of II-VI compounds and $(n, n)$ and $(n, 0)$ CNTs, but also to formulate the appropriate carrier statistics in the most generalized form, since the transport and other phenomena in semiconductors having different band structures and the derivation of the expressions of many important electronic properties are based on the temperature-dependent electron statistics in such materials.

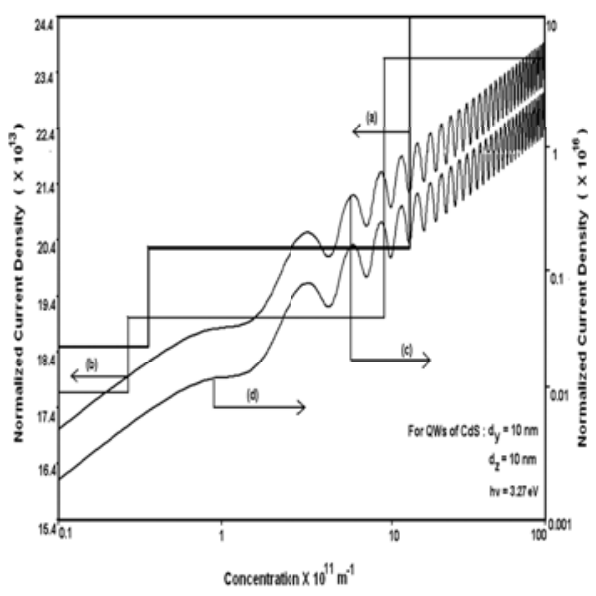

Fig.1. Plot of the normalized photoemission as functions of concentration from QWs of CdS in which $c_{0} \quad 0$ (a) and (b) $\mathrm{C}_{0}=0$ and CNTs in which (c) $(n, n)$ CNT and (d) $(n, 0)$ CNT. 


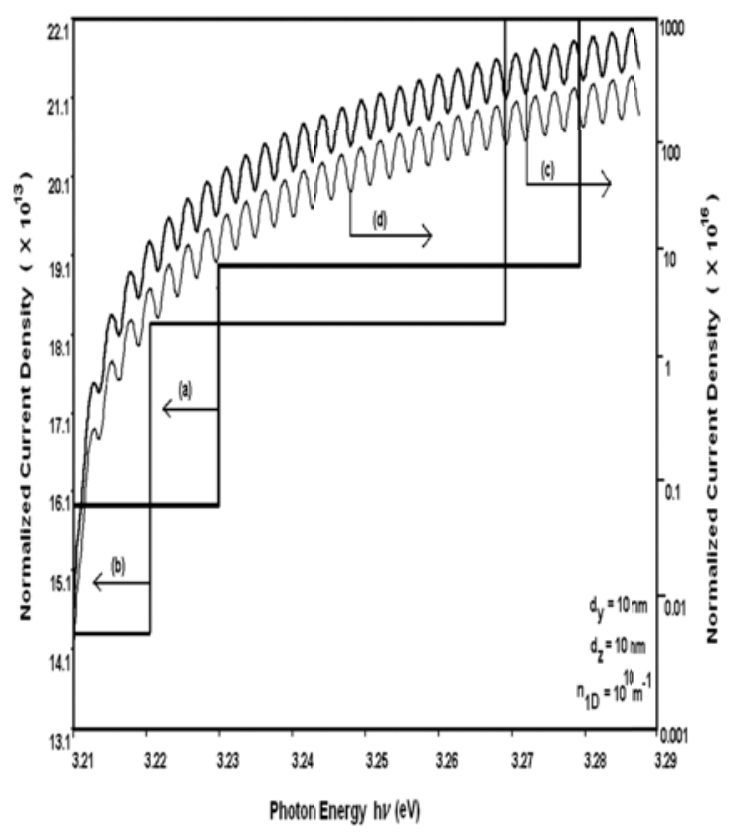

Fig.2. Plot of the normalized photoemission as functions of incident photon energy from $\mathrm{QWs}$ of $\mathrm{CdS}$ in which $c_{0} \quad O(a)$ and $c_{0}=0(b)$ and CNTs in which $(c)(n, n)$ CNT and $(d)(n, 0)$ CNT.

\section{REFERENCES}

[1] J. W. Elam, J. A. Libera, M. J. Pellin, and P. C. Stair, 2007, "Spatially controlled atomic layer deposition in porous materials," Appl. Phys. Lett. USA, 91, pp. 243105-243108.

[2] L. L.Chang, H.Esaki, C.A. Chang, and L.Esaki, 1977, "Shubnikov-de Haas Oscillations in a Semiconductor Superlattice," Phys. Rev. Lett. USA, 38, pp.1489-1493.

[3] Y.Arakawa and H. Sakaki, 1982, "Multidimensional quantum well laser and temperature dependence of its threshold current," Appl. Phys. Lett. USA, 40, pp.939-941.

[4] K. Akita, R. Akimoto, T. Hasama, H. Ishikawa and Y. Takanashi, 2006, "Intersubband all-optical switching in submicron high-mesa SCH waveguide structure with wide-gap II-VI-based quantum wells," IEE Electronics Letts, USA, 42, pp. 13521353.

[5] J. J. Hopfield, 1961, "Exciton States and Band Structure in CdS and CdSe," J. Appl. Phys. USA, 32, pp. 2277-2281.
[6] K. C. Lin, H. W.Chen, C. P.Juan, R.L. Lai, Y.Y. Hsu, and H.C. Cheng, 2008, "Carbon-Nanotube-Based Field Emission Devices with a Self-Focusing Gate Structure," J. Electrochem. Soc. USA, 155, pp. K38-K41.

[7] G. D. Hatchel and A. E. Ruchli, 1968, "The charge distribution in high-speed transistors," IEEE Trans. Electron. Dev. USA, 15, pp. 437-437.

[8] A.Van Der Ziel, 1957, Solid State Physical Electronics, , New York, Prentice-Hall.

[9] X. Yang and J. Ni, 2005, "Electronic properties of single-walled silicon nanotubes compared to carbon nanotubes," Phys. Rev. B. USA, 72, pp. 195426-195431.

[10] O. Madelung, 2004Semiconductors: Data handbook, Third Edition, Springer.

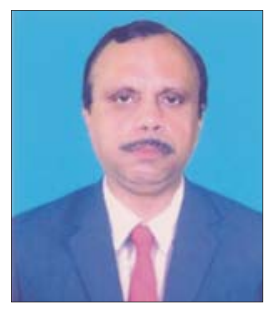

Dr. SHANKAR NATH BANERJEE has completed B.E. degree in Electronics and Communication and M.E degree from Calcutta University,Sibpur, India in 1978 \& 1980 respectively $\mathrm{He}$ has Completed his Phd in the year 2001 at Jadavpur University, India. $\mathrm{He}$ is presently working as Joint Director (Instrumentation) in I.T.R., Chandipur. He have contributed in the areas of augmentation, indigenisation and design and development of Range Instrument Systems.He has published more than 15 papers in International Conferences and Journal. 